\title{
Tear lipid layer thickness with eye drops in meibomian gland dysfunction
}

This article was published in the following Dove Press journal:

Clinical Ophthalmology

7 November 2016

Number of times this article has been viewed

Jennifer S Fogt'

Matthew J Kowalski'

P Ewen King-Smith'

Alice T Epitropolous ${ }^{2}$

Andrew J Hendershot ${ }^{2}$

Carrie Lembach ${ }^{2}$

John Paul Maszczak'

Lisa A Jones-Jordan'

Joseph T Barr'

'The Ohio State University College of Optometry, ${ }^{2}$ The Ohio

State University Department of

Ophthalmology, Columbus, $\mathrm{OH}$, USA

Video abstract

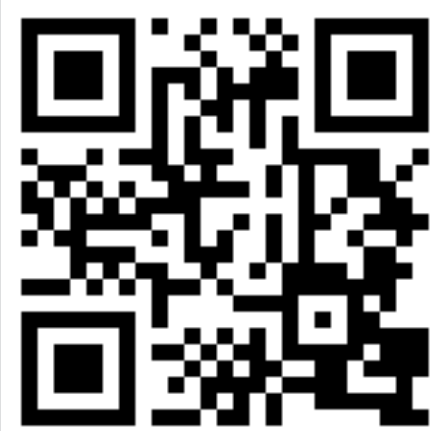

Point your SmartPhone at the code above. If you have a QR code reader the video abstract will appear. Or use: http://youtu.be/NwVl|x40aA8s

Correspondence: Jennifer S Fogt The Ohio State University College of Optometry, 338 W I0th Ave, Columbus, $\mathrm{OH} 43210$, USA

Tel +l 6146884594

$\mathrm{Fax}+\mathrm{I} 6146885603$

Email fogt.78@osu.edu
Purpose: The aim of this study was to evaluate the efficacy of a lipid containing emollient eye drop, Soothe XP, which was reformulated in 2014 with a more stable preservative and buffer system, compared to a control, non-emollient, eye drop (Systane Ultra) in improving lipid layer thickness (LLT) in subjects with dry eye due to meibomian gland dysfunction (MGD).

Patients and methods: This prospective single-center, open-label, cross-over, examiner masked-study enrolled subjects aged 30-75 years with lipid-deficient dry eye and a clinical diagnosis of MGD as determined by a slit lamp examination, an evaluation of meibomian gland drop out with meibography, and a standard patient evaluation of eye dryness questionnaire of $>5$. Eligibility was then determined by a LLT of $<75 \mathrm{~nm}$ at baseline and the inability to increase LLT $\geq 15 \mathrm{~nm}$ with three blinks, as determined by interferometric methods. Subjects were randomized to receive a single emollient or non-emollient eye drop at Visit 1 and were crossed over for the alternate treatment at Visit 2. At each visit, LLT was measured prior to and 15 minutes following the instillation of the assigned eye drop. The primary endpoint was the change in LLT from baseline.

Results: Subjects $(n=40)$ were enrolled and 35 completed the two study arms. Mean $( \pm S D)$ patient age was 55.7 years $(10.9)$ and $69 \%$ were female. Mean $( \pm$ SD) LLT at baseline was $49.5 \mathrm{~nm}$ (9.2). Instillation of Soothe XP resulted in an increase in LLT to $77.5 \mathrm{~nm}(29.3)$ 15 minutes following drop instillation, which is an increase of $28.0 \mathrm{~nm}(27.4)(P<0.001)$. In contrast, LLT 15 minutes after the instillation of Systane Ultra was $50.8 \mathrm{~nm}$ (14.1), which was not statistically significant when compared to the baseline LLT.

Conclusion: In this study of subjects with MGD, the emollient, or lipid containing eye drop, increased the LLT of tears when measured 15 minutes after instilling a single eye drop.

Keywords: dry eye, lipid, emollient, thickness, meibomian, interferometry

\section{Introduction}

Dry eye is one of the most common ocular disorders and has been estimated to have a societal cost of $\$ 55$ billion per annum in the US. ${ }^{1}$ The prevalence of dry eye varies based on parameters used to gather data and ranges from $14 \%$ for patients over age 48 in the US, $25 \%$ in Canada, and 33\% in Taiwan and Japan. ${ }^{2}$

Many aspects of dry eye are still not well understood and its management can be challenging. Dry eye can be the result of inadequate aqueous tears being produced by the lacrimal gland or meibomian gland dysfunction (MGD), which results in evaporation of the tear film. ${ }^{3}$ Lipid deficiency due to MGD is the most common cause of symptoms associated with dry eye disease. ${ }^{4}$ The meibomian glands in the eyelids secrete meibum, a lipid complex that forms the lipid layer of the tear film. The lipid layer prevents evaporation of aqueous tears and subsequent drying. This causes increased tear osmolarity which in turn can lead to ocular surface inflammation and damage. ${ }^{5}$ Over-the-counter 
(OTC) artificial tear supplementation is commonly used for the relief of dry eye symptoms. While the extent to which tear evaporation changes with the application of lipid containing drops is not well established; ${ }^{6}$ clinicians recommend drops with lipid to enhance the lipid layer of MGD patients for relief of dry eye symptoms. There is increased interest in MGD following the report of the International Workshop that was published in 2011.4

Korb et al have previously shown that the eye drop Soothe, an emollient which contains mineral oil, can increase the lipid layer of the tear film from $\sim 75$ to $125 \mathrm{~nm}$. Soothe $^{7}$ was later acquired by Bausch+Lomb and the emollient drop was renamed Soothe XP. Bausch+Lomb reformulated the eye drop in 2014 with a more stable preservative and buffer system, keeping the active ingredients and all other excipients unchanged. While the performance of the reformulated eye drop is expected to be the same as the original formulation, no study has yet been done to show if the tear lipid layer thickness (LLT) is increased with the new eye drop.

An improved understanding of the sequence of events leading to ocular surface inflammation and damage requires study of both the characteristics of the lipid layer and the consequent changes to the ocular surface and in the composition of the aqueous tears. Optical studies can provide important information about the dry eye, namely the changes in the lipid layer which may cause evaporative dry eye. King-Smith et al have developed optical methods for studying the tear film and the lipid layer, based on both the self-quenching of fluorescence and optical interference in the tear film, which provide unique information about tear film structure, function, and dynamics. ${ }^{8}$

A recent study by King-Smith showed that the tear lipid layer structure is fluid and changing constantly as a result of the blink cycle. ${ }^{9}$ Using interferometric methods, the thickness of the lipid layer was measured and viewed as it moved over the surface of the eye. In healthy eyes, this apparent irregularity in the tear film is not believed to affect vision, and the presence of rippling, which appears in both the upstroke and downstroke of a blink, is considered normal. ${ }^{9}$ Of greater clinical concern, it is thought that the presence of rippling is disruptive in an eye which has a thinner tear film, in which case it may cause significant thickness variation and tear break up, which in turn could decrease vision. ${ }^{9}$

The purpose of this study was to evaluate the efficacy of reformulated Soothe XP (Bausch+Lomb) in improving LLT in subjects with dry eye symptoms, MGD, and lipid layer deficiency (LLT $<75 \mathrm{~nm}$ at baseline), similar to the study design used by Korb et al in $2005 .^{7}$

\section{Materials and methods}

This was a prospective, cross-over, examiner masked study of the change in LLT in subjects with MGD and lipid deficiency following instillation of a single drop of either new Soothe XP or a single drop of Systane Ultra (Alcon Laboratories, Inc, Fort Worth, TX, USA), a non-emollient eye drop which was used as a control. The study was completed under the approval of the Institutional Review Board at The Ohio State University, located in Columbus, Ohio, USA. Subjects provided written informed consent prior to screening.

The present study was designed as similar as possible to the design used by Korb in his 2005 paper. ${ }^{7}$ Our methods of measuring LLT on normal eyes are similar to those of Korb, although our interferometric measurements were acquired with a different device. The device we used for interferometric evaluation of the LLT is a stroboscopic video color microscope (SVCM) developed by King-Smith and used to study tear film dynamics with Braun et al. ${ }^{9}$ This instrument allows the visualization of the lipid layer and is calibrated to measure its thickness to the nanometer $(\times 10$ to $\times 100 \mathrm{~nm}$ range). Images at different phases of the blink cycle can be recorded. ${ }^{9,10}$ The microscope has a stroboscopic, white light source that covers a circular area of $6 \mathrm{~mm}$ diameter and uses a high performance color camera $(1,400 \times 1,100$ pixels, 22 images/sec, and flash duration $0.04 \mathrm{msec}$ ). The camera records a video image which is comprised of 900 frames. Software gives a quality assessment based upon the number of those pixels actually used in a given calculation, giving an assessment of the validity of the measurement. The thickness of all of these data points is used to give the overall LLT measurement for the segment. Video image analysis was performed by software designed by King-Smith to convert each pixel of the images into an LLT measurement in nanometers. Blink disruption of the continuous data is deleted.

The study consisted of a prescreening visit to determine the presence of MGD and therefore, eligibility; a screening visit in which baseline (LLT) was measured with SVCM, and two study visits to measure the LLT both before instilling a single randomized eye drop, and again 15 minutes after the instillation. The eye drop visits were scheduled on different days to ensure washout.

\section{Subjects}

The study enrolled subjects aged 30-75 years with tear lipid deficiency and symptomatic dry eye disease. Potential subjects were either pre-screened for the study at an exam given by their eye doctor, or answered an advertisement and made an appointment for an eligibility visit. This evaluation included an eye screening with a biomicroscope, in which the following were evaluated: lack of obvious normal 
meibum with 3 forced blinks, corneal/conjunctival staining, visible meibomian gland orifice capping, and abnormal/ inspissated meibomian gland secretion when pressure was applied to the lid. Meibomian gland drop out was assessed with meibography. ${ }^{11}$ A score of 5 or greater on the one page standard patient evaluation of eye dryness questionnaire ${ }^{12}$ was required. Exclusion criteria included: any current treatment of MGD; any use of prescription or OTC eye drops; current eye infection or inflammation; full-time contact lens wear; females could not be pregnant or lactating; active systemic or ocular infectious disease; or anyone who was not able to use the equipment due to size or inability to hold fixation of the eye for 1 minute. An additional exclusion was a history of eye surgery, with the exception of cataract removal surgery at least 1 year, but not $>10$ years prior, which was an exclusion in the previous Soothe XP study done by Korb et al. ${ }^{7}$

Those subjects eligible were consented and scheduled for a screening visit. On the screening and measurement visit days, to participate in the study, subjects were directed to refrain from eye rubbing prior to and during the assessment and study visit and to refrain from using eye makeup, similar to the protocol followed by Korb et al.

The purpose of the screening visit was to determine if the subject was eligible to participate in the eye drop portion of the study. In order to be eligible for the drop portion, subjects were required to have a LLT of $75 \mathrm{~nm}$ or less, with an increase of no $>15 \mathrm{~nm}$ after a set of three blinks (Figure 1).

Room temperature was controlled at $25^{\circ} \mathrm{C}( \pm 1)$ and relative humidity at 35\%-50\%.

To obtain LLT measurements, subjects were seated in front of the SVCM and instructed to place their chin in a chin rest and press their forehead against a forehead rest for stabilization. The instrument allowed for measurement with minimal subject adjustment needed.

The subject was given verbal instructions prior to and during the measurement period including where to look, and when to blink. The test itself lasted $<1$ minute per eye.

Those patients who were found to be eligible for the eye drop portion of the study were randomly assigned to one of the eye drops based on a randomization list prepared by the statistician. LLT was measured by SVCM prior to and 15 minutes following instillation of the assigned drop into the inferior cul-de-sac, being careful not to apply pressure to the meibomian glands. A new bottle of each eye drop was opened for each subject and shaken vigorously up and down 20 times prior to instillation of a single drop. The subject was asked to blink normally after the drop was instilled, only blotting excess drop from his/her face gently, without any eyelid rubbing or excessive blinking until the 15-minute post-installation LLT measurements were completed.

For the eye drop visits, the SVCM measurement was taken with the subject being instructed to blink every 4 seconds, in order to replicate the normal blinking rate ${ }^{13}$ and prevent the subject from staring into the light of the SVCM. Fifteen minutes after the eye drop was instilled, this measurement was repeated with the investigator again prompting the subject to blink at 4-second intervals.

Subjects returned to the clinic at Visit 2 in which all procedures were repeated using the alternative eye drop. The two eye drop test visits were scheduled on separate days to ensure a wash-out between the drops.

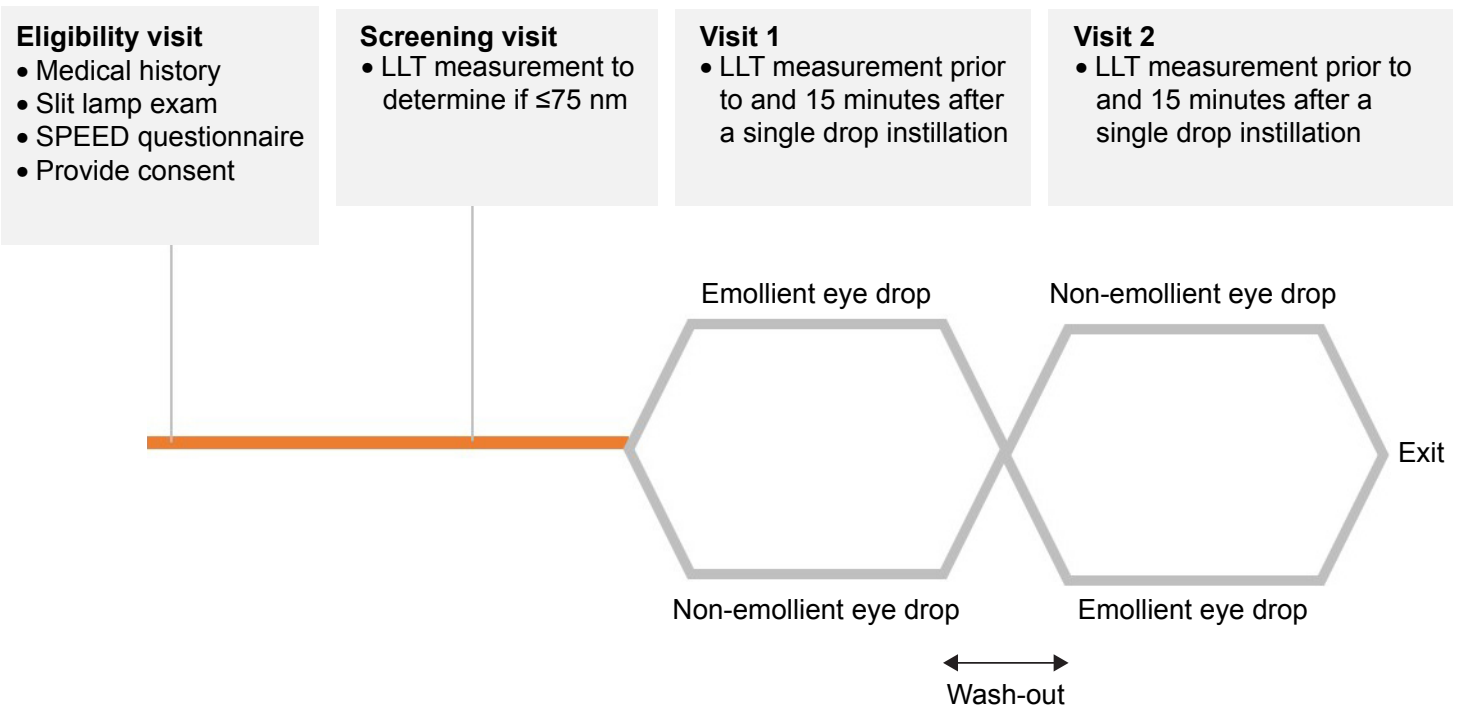

Figure I Study design.

Abbreviations: LLT, lipid layer thickness; SPEED, standard patient evaluation of eye dryness. 
The same eye drops were used in both eyes for each measurement visit. For subjects whose screening visit identified only one eye as eligible, this eye was used for analysis, but both eyes were measured at all visits. Although it was impossible to mask the investigator from seeing which drops were instilled, the video file collected was masked upon collection. Data analysis was done by computer and the eye drop used for each test was not revealed until after data analysis for all subjects was complete.

\section{Test articles}

Soothe XP (Soothe XP-Xtra Protection Emollient [Lubricant] Eye Drops, Bausch+Lomb) contains light mineral oil (1.0\%) and mineral oil (4.5\%) as lower and higher molecular weight emollients and boric acid, edetate disodium, octoxynol-40, polyquaternium-1, polysorbate 80 , purified water, and sodium borate decahydrate as inactive ingredients. Systane Ultra (Systane ${ }^{\circledR}$ Ultra Lubricant Eye Drops, Alcon Laboratories, Inc) contains polyethylene glycol $400(0.4 \%)$ and propylene glycol $(0.3 \%)$ as lubricants and aminomethylpropanol, boric acid, hydroxypropyl guar, polyquaternium-1, potassium chloride, purified water, sodium chloride, and sorbitol as inactive ingredients. Both products may contain hydrochloric acid and/or sodium hydroxide to adjust $\mathrm{pH}$.

\section{Statistical analysis}

The subject sample size was based on a previous study done by Korb et al, ${ }^{7}$ which found a baseline mean $( \pm \mathrm{SD})$ LLT of 60 (1.8) and a "treatment effect" of increased LLT to $125 \mathrm{~nm}$ for Soothe XP and to $70 \mathrm{~nm}$ for Systane Ultra at 15 minutes after instillation.

The LLT data analysis was based on the bottom third of this circular area captured, which had 229,000 pixels capable of collecting data. The thickness of all data points in this area is averaged to provide a LLT measurement for the segment.

The subject's study eye was either: the only eye that qualified; or the eye that had the thinnest LLT (ie, worst eye) at the baseline qualifying visit if both eyes qualified. Withinsubject changes from the baseline pre-blink lipid layer were calculated for each drop and compared to 0 to determine if a significant change had occurred via paired $t$-test. Likewise, the change from baseline (CFB) for the emollient eye drop was compared to the CFB for the non-emollient eye drop using a paired $t$-test. Differences among baseline measurements at each of the visits were assessed using a repeated measures model. A $P$-value $<0.05$ was considered significant. All analyses were completed using SAS 9.3 software by SAS Analytics.

\section{Results}

Of the 64 subjects screened, 40 were eligible to enter the study. Five were immediately disqualified either due to difficulty fitting into the equipment, inability to fixate the eye, or inability to keep eyes, head, or body still for measurements. Of the remaining 35 subjects, all 35 completed the two study arms. The mean $( \pm \mathrm{SD})$ age of these subjects was 55.7 years (10.9) and $69 \%$ were female. Five of the 35 subjects had only one eligible eye based on LLT. Mean $( \pm$ SD) LLT at the baseline qualifying visit was 49.5 (9.2) $\mathrm{nm}$ for study eyes.

The baseline mean $( \pm \mathrm{SD})$ LLT measurement obtained at the qualifying visit was $49.5 \mathrm{~nm}$ (9.2) in study eyes. Additional pre-drop LLT measurements were taken at the beginning of each randomized eye drop visit, giving a total of three LLT measurements obtained without the use of any eye drops. The LLT was $49.1 \mathrm{~nm}$ (10.8) before an eye drop was instilled for study eyes at the randomized visit for the emollient eye drop and $50.2 \mathrm{~nm}$ (14.1) study eyes when at the randomized visit for the non-emollient instillation. These additional measurements did not differ from mean LLT in study eyes at the qualifying baseline visit $(P>0.05)$ (Figure 2).

Instillation of the emollient eye drop led to a significant increase in mean ( \pm SD) LLT to $77.5 \mathrm{~nm}$ (29.3) at 15 minutes post-instillation. The mean CFB was $28.04 \mathrm{~nm}$ (27.4); $P<0.001$. Instillation of the non-emollient eye drop had no significant impact on LLT 15 minutes following instillation, mean $( \pm \mathrm{SD}) \mathrm{LLT}$ was $50.8 \mathrm{~nm}(14.1)$ (mean [ $\pm \mathrm{SD}] \mathrm{CFB}$ of $1.4 \mathrm{~nm}[10.7] ; P=0.6)$.

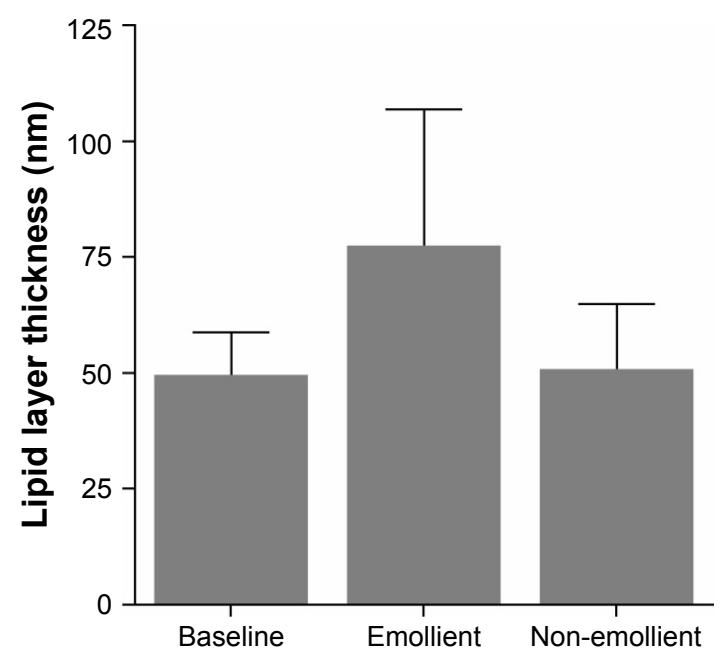

Figure 2 LLT prior to and I 5 minutes following a single drop of emollient or nonemollient eye drop in dry eyes with meibomian gland dysfunction. Data are the mean $( \pm S D)$ LLT based on stroboscopic video color microscope (SVCM) measurements in study eyes (qualifying eye in subjects with only one qualifying eye, or the eye with the lowest LLT at baseline in subjects with two qualifying eyes). $P<0.00$ I paired $t$-test for the change from baseline.

Abbreviation: LLT, lipid layer thickness. 


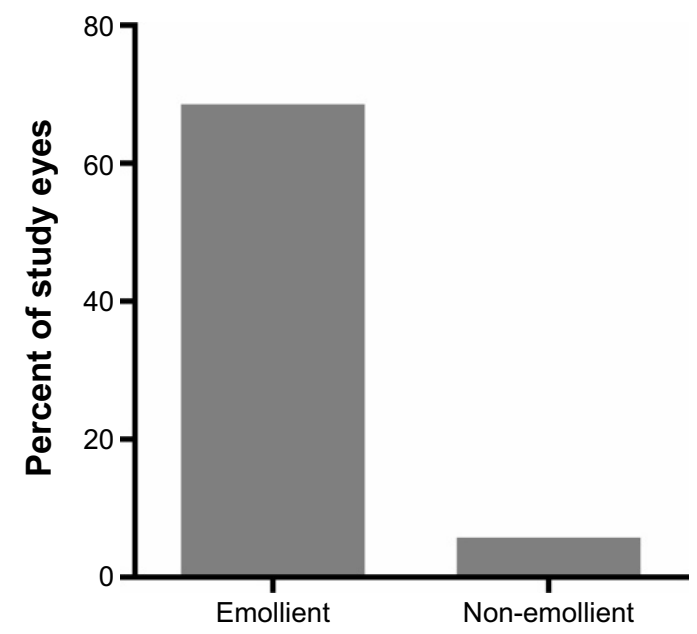

Figure 3 Percentage of study eyes with a clinically significant increase in lipid layer thickness 15 minutes following a single drop of emollient or non-emollient eye drop in dry eyes with meibomian gland dysfunction. A clinically significant increase was defined as an increase of $\geq 15 \mathrm{~nm}$ from baseline as determined by stroboscopic video color microscope interferometry.

Note: $P<0.00$ I chi-square test, two-tailed for the difference in percentages.

Overall, 94\% (33/35) of study eyes had an increase of any amount in LLT with the emollient eye drop compared to $49 \%$ (17/35) with the non-emollient eye drop $(P<0.001$ for the difference in proportions). Using $\mathrm{a} \geq 15 \mathrm{~nm}$ change to define a clinically significant change (based on the eligibility criteria used in determining subject eligibility in our study, which is also used by Korb et al $\left.{ }^{7}\right), 68 \%$ (24/35) of study eyes had a clinically significant increase in LLT following instillation of the emollient, whereas none had a clinically significant decrease in LLT. In contrast, $2.9 \%(1 / 35)$ of study eyes had a clinically significant increase and decrease in LLT following instillation of the non-emollient eye drop (Figure 3). The difference between treatments in the proportion of study eyes with a clinically significant increase in LLT was also statistically significant $(P<0.001)$.

Figure 4 depicts representative interference images of the lipid layer of the tears captured at the baseline qualifying visit
(Figure 4A), and at each of the eye drop visits 15 minutes following instillation of a single drop of the emollient eye drop (Figure 4B) or the non-emollient eye drop (Figure 4C). In these images, a lack of color is representative of a thin lipid layer, whereas colors represent a thicker lipid layer as noted by the color scale on each image. Fifteen minutes following the instillation of the emollient, there was a notable increase in color (changed from gray) with large amounts of brown interference fringes in the image. In contrast, 15 minutes after the instillation of the non-emollient, the image was nearly identical to the gray seen in the baseline image, demonstrating that the LLT was not altered through the use of our control, non-emollient eye drop.

\section{Discussion}

The purpose of this study was to measure the change in LLT following instillation of reformulated Soothe XP, an emollient eye drop, and compare it to the change in LLT after instillation of a non-emollient eye drop in the same subjects who have MGD. Consistent with a previous study on the original formulation of Soothe XP conducted by Korb et al, ${ }^{7}$ there was a statistically significant increase in LLT after instillation of the emollient eye drop, whereas we found no change after instillation of the non-emollient eye drop. Of note, there were quantitative differences in all LLT measurements between our study and the study by Korb et al. ${ }^{7}$ In our findings, the emollient eye drop gives an average $58 \%$ increase in the LLT of the tear film, whereas Korb et al reported a much greater increase in LLT (107\%) following instillation of the original formulation of the emollient eye drop and a small (16\%) increase following instillation of the non-emollient.

While the eligibility criteria for this study and the previous study were identical, there were differences in the designs of the device used to measure LLT. For instance, the exact area of the tear film in which the LLT was measured was
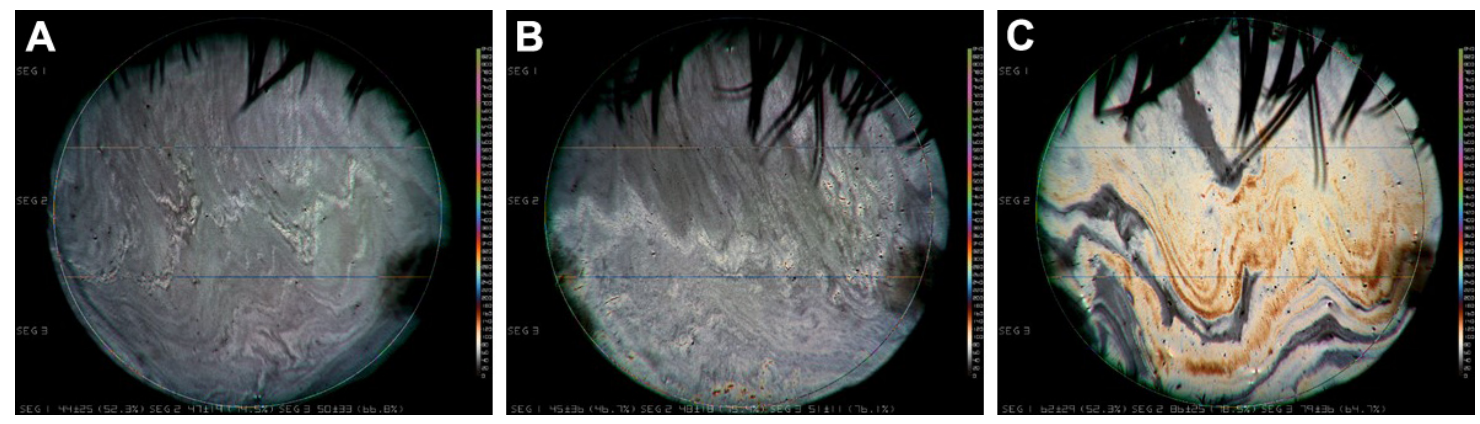

Figure 4 Representative stroboscopic video color microscope (SVCM) images of the lipid layer of the tears at the baseline qualifying visit (A); 15 minutes following instillation of a single drop of a non-emollient eye drop (B); and 15 minutes following the instillation of a lipid containing (emollient) eye drop (C).

Note: Note that gray, or a lack of color, is representative of a thin lipid layer, whereas bright colors represent thicker lipid layers, as noted in the color scale beside each image. 
different between studies. In the previous study, the area of the LLT measured was described as $\sim 2.5 \mathrm{~mm}$ in height and $5 \mathrm{~mm}$ in width, with the bottom of the area $\sim 1 \mathrm{~mm}$ above the inferior meniscus. ${ }^{7}$ The SVCM measures the LLT of a $6 \mathrm{~mm}$ diameter circular area centered in front of the pupil. In an attempt to measure LLT over a similar area to that of the previous study by Korb et al, the circular area measured by our method was divided into thirds as shown by the horizontal lines in the images of Figure 4, with the lower third of each image being the area used for data collection. While these two areas may not be identical, they share a substantial overlap in the area of the lipid layer measured.

Additional differences could be due to the method of obtaining the LLT data. In our study, the SVCM images were analyzed for LLT objectively, ${ }^{14}$ using the analysis software by computer. Baseline and two pre-drop LLT measurements were nearly identical, showing repeatability when using SVCM as an interferometric method of measuring LTT. The previous study used a method of grading the colors of the images produced by the interferometer. In that method, two independent observers graded the thickness of the lipid layer by judging the dominant color present in the interference pattern image created by the instrument, and used a chart to obtain the LLT based upon this dominant color. This differs from an observation system in which one color had to be chosen to represent the entire area. This difference in methodology was expected to give us lower LLT values, as can be seen both by the fact that many gray, "thinner" patches are often present. This difference is evident when comparing the change in LLT with the use of the control, the non-emollient eye drop, which does not contain a lipid. In the previous study, the non-emollient provided an increase in LLT, while there was no increase in the current study as measured with SVCM, as expected. Therefore, it would be expected that the LLT measurement increase for the emollient eye drop would also be lower than measured by Korb et al. ${ }^{7}$ It appears that the lower LLT increase in this study is related to the fact that the instrumentation and techniques used in this study are somewhat different.

\section{Conclusion}

In this study there was a statistically significant increase in LLT after instillation of the emollient eye drop, whereas we found no change in the LLT after instillation of the nonemollient eye drop. This is consistent with a previous study done with an earlier formulation of the same lipid-containing eye drop. ${ }^{7}$ Although there is an evidence that factors other than the LLT play a role in tear film thinning in evaporation, ${ }^{15}$ lipid containing eye drops that increase LLT to a statistically or clinically significant level may assist MGD patients. A significant increase in LLT may provide practitioners with a valuable tool in giving their patients temporary relief from the discomfort of dry eye. Further studies of the lipid layer are essential to better understand dry eye.

\section{Acknowledgments}

This research was funded by Valeant Pharmaceuticals North America LLC. The authors would like to thank Rebecca Kuennen for helpful comments and thank Jeffery Fardink for his assistance in completing our data base for this study. This study was presented in part at the Association for Research in Vision and Ophthalmology meeting in Seattle, Washington, May 5, 2016.

\section{Disclosure}

Jennifer S Fogt and Alice T Epitropoulos are speakers and consultants for Bausch+Lomb. Joseph T Barr is a consultant for Bausch+Lomb. The authors report no other conflicts of interest in this work.

\section{References}

1. Yu J, Asche CV, Fairchild CJ. The economic burden of dry eye disease in the United States: a decision tree analysis. Cornea. 2011;30(4): 379-387.

2. Gayton JL. Etiology, prevalence, and treatment of dry eye disease. Clin Ophthalmol. 2009;3:405-412.

3. Lemp MA, Crews LA, Bron AJ, Foulks GN, Sullivan BD. Distribution of aqueous-deficient and evaporative dry eye in a clinic-based patient cohort: a retrospective study. Cornea. 2012;31(5):472-478.

4. Nichols KK, Foulks GN, Bron AJ, et al. The international workshop on meibomian gland dysfunction: executive summary. Invest Ophthalmol Vis Sci. 2011;52(4):1922-1929.

5. Braun RJ, Gewecke NR, Begley CG, King-Smith PE, Siddique JI. A model for tear film thinning with osmolarity and fluorescein. Invest Ophthalmol Vis Sci. 2014;55(2):1133-1142.

6. Kimball SH, King-Smith PE, Nichols JJ. Evidence for the major contribution of evaporation to tear film thinning between blinks. Invest Ophthalmol Vis Sci. 2010;51(12):6294-6297.

7. Korb DR, Scaffidi RC, Greiner JV, et al. The effect of two novel lubricant eye drops on tear film lipid layer thickness in subjects with dry eye symptoms. Optom Vis Sci. 2005;82(7):594-601.

8. King-Smith PE, Fink BA, Fogt N. Three interferometric methods for measuring the thickness of layers of the tear film. Optom Vis Sci. 1999; 76(1):19-32.

9. Braun RJ, King-Smith PE, Begley CG, Li L, Gewecke NR. Dynamics and function of the tear film in relation to the blink cycle. Prog Retin Eye Res. 2015;45:132-164.

10. Doane MG. An instrument for in vivo tear film interferometry. Optom Vis Sci. 1989;66(6):383-388.

11. Pult H, Riede-Pult B. Comparison of subjective grading and objective assessment in meibography. Cont Lens Anterior Eye. 2013;36(1): 22-27.

12. Ngo W, Situ P, Keir N, Korb D, Blackie C, Simpson T. Psychometric properties and validation of the standard patient evaluation of eye dryness questionnaire. Cornea. 2013;32(9):1204-1210. 
13. Bentivoglio AR, Bressman SB, Cassetta E, Carretta D, Tonali P, Albanese A. Analysis of blink rate patterns in normal subjects. Mov Disord. 1997;12(6):1028-1034.

14. Millar TJ, King-Smith PE. Analysis of comparison of human meibomian lipid films and mixtures with cholesteryl esters in vitro films using high resolution color microscopy. Invest Ophthalmol Vis Sci. 2012;53(8): 4710-4719.
15. Guillon JP. Abnormal lipid layers. Observation, differential diagnosis, and classification. Adv Exp Med Biol. 1998;438:309-313.

\section{Publish your work in this journal}

Clinical Ophthalmology is an international, peer-reviewed journal covering all subspecialties within ophthalmology. Key topics include: Optometry; Visual science; Pharmacology and drug therapy in eye diseases; Basic Sciences; Primary and Secondary eye care; Patien Safety and Quality of Care Improvements. This journal is indexed on

Submit your manuscript here: http://www.dovepress.com/clinical-ophthalmology-journal

\section{Dovepress}

PubMed Central and CAS, and is the official journal of The Society of Clinical Ophthalmology (SCO). The manuscript management system is completely online and includes a very quick and fair peer-review system, which is all easy to use. Visit http://www.dovepress.com/ testimonials.php to read real quotes from published authors. 